\title{
A Course in Writing \& Appraising Medical Papers
}

\section{Theodora Benedek}

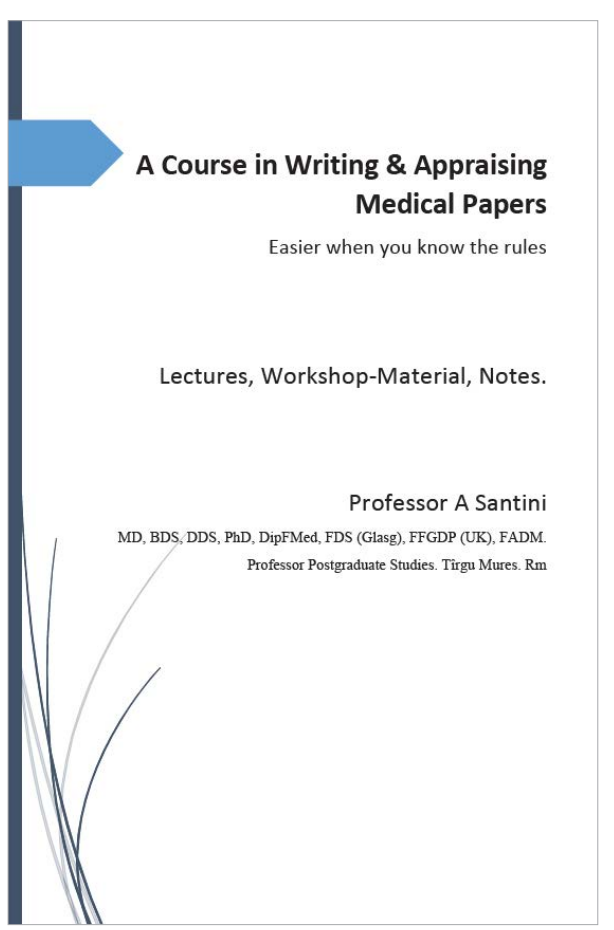

Book title

A Course in Writing \& Appraising Medical Papers - Easier when you know the rules

\section{Author}

Professor Ario Santini
The book entitled „A Course in Writing \& Appraising Medical Papers Easier when you know the rules" is a comprehensive work which contains information on how to improve the understanding and also the technique in writing and submitting a scientific paper to specialized medical journals. The book represents a written support for an online course on how to write a medical paper. The manuscript together with the online course aims to improve the reader's understanding on the format of a scientific paper, on how to avoid manuscript rejection, on how the peer-review process is functioning, and also on the ethical concepts in the medical publication industry. The text also contains information on different study designs, their strong points and drawbacks.

The book is structured in 4 parts. The first part contains several lectures for an online course, each lecture tackling different steps that are to be followed when writing a medical research paper, such as: planning a paper, the abstract writing, the structure of different types of articles (case report, review, or letter to the editor), statistical analysis, and common mistakes that are made in the process of designing a medical article. The second part contains information that can sustain the workshop sessions on Critical Appraisal. At the end of the second part, there is a paper on which the participants can practice their critical appraise. In the third section, each part of the paper that was shown at the end of the 2nd part are commented, including written advice on each segment. The fourth part on the manual includes a list of answers to frequently asked questions during previous sessions of the online course.

The overall structure of the book is based on a complex approach on the process of writing a scientific paper, and it is of high importance for medical researchers and for any person participating in the designing, writing and publishing a research thesis. The integration of all the related information on the topic, associated with the online course, makes this a manuscript of high value to those who seek to learn the best approach in designing and creating a scientific research study. The comprehensive approach on the matter gives the reader a chance to improve their knowledge in how to write and submit a medical article in recognized scientific journals. 
All the presented features from above show that the manual, in relation with the online course, gives the reader extremely usefull tools regarding the scientific research methodology and the basic concepts and steps that need to be followed in order to elaborate a high quality reaserch paper. 